\title{
Time based readout of a silicon photomultiplier (SiPM) for Time Of Flight Positron Emission Tomography (TOF-PET)
}

\author{
P. Jarron(IEEE member), E. Auffray(IEEE member), S.E. Brunner, M. Despeisse, E. Garutti, M. Goettlich, \\ H. Hillemanns, P. Lecoq (IEEE member), T. Meyer, F. Powolny, W. Shen, H.C. Schultz-Coulon, C. Williams
}

\begin{abstract}
Time of flight (TOF) measurements in positron emission tomography (PET) are very challenging in terms of timing performance, and should achieve ideally less than 100ps FWHM precision. We present a time-based differential technique to read out SiPMs that has less than 25ps rms electronic jitter. The novel readout is a fast front end circuit (NINO) based on a first stage differential current mode amplifier with $20 \Omega$ input resistance. Therefore the amplifier inputs are connected differentially to the SiPM's anode and cathode ports. The leading edge of the output signal provides the time information, while the trailing edge provides the energy information. Based on a Monte Carlo photon-generation model, SPICE simulations were run with a $3 \times 3 m^{2}$ SiPM-model, read out with a differential current amplifier. The results of these simulations are presented here and compared with experimental data obtained with a $3 \times 3 \times 15 \mathrm{~mm}^{3}$ LSO crystal coupled to a SiPM. The measured time coincidence precision is interpreted by the combined Monte Carlo/ SPICE simulation, as well as by Poisson statistics.
\end{abstract}

\section{INTRODUCTION}

$\mathrm{T}^{\mathrm{s}}$ he time of flight technique applied in PET could make a major improvement in sensitivity over standard PET methods [1]. So far TOF-PET detector heads have been implemented using PMTs, and the next step would be the replacement of the PMT with a silicon photomultiplier (SiPM) [2]. SiPMs offer obvious advantages in terms of compactness and tolerance to magnetic field, with an energy resolution comparable to the PMT. However, the time precision of a PET channel built with a LSO crystal and SiPM looks so far inferior to a PMT-equipped channel. Our paper aims to discuss a fast front end electronics system that could be the building block of a readout architecture for a multichannel ASIC in a TOF-PET detector. It also tries to identify the parameters limiting the ultimate timing precision achievable in a SiPM-PET detector channel. In particular our work investigates the limits in timing precision imposed by the

Manuscript received November 4, 2009.

P. Jarron, E. Auffray, S.E. Brunner, H. Hillemanns, P. Lecoq, T. Meyer, F. Powolny are with CERN, CH-1211 Geneva 23, Switzerland, (e-mail: pierre.jarron@cern.ch).

C. Williams is with the University of Bologna, Via Zamboni, 33 - 40126 Bologna Italy

M. Despeisse is with Ecole Polytechnique Federale de Lausanne EPFL/STI, Rue A.L Breguet 2, 2000 Neuchatel, Switzerland

E. Garutti, M. Goettlich are with Deutsches Elektronen-Synchrotron DESY, Notkestraße 8522607 Hamburg - Germany.

H.C. Schultz is with the University of Heidelberg, Kirchhoff Institute for Physics, Im Neuenheimer Feld 227, D-69120 Heidelberg readout electronics, the photon emission and the light transport in the scintillating crystal, and by Poisson statistics. Monte Carlo simulation is also used to generate sequences of random photons impinging on the SiPM, whereas SPICE is used to study the entire readout channel response. Both comprise the photon generation in the scintillator and electronic signal processing from the SiPM to the output of the differential amplifier-discriminator.

SiPM readout is so far configured with a single-ended connection to the front end amplifier inputs [3, 4]. In this work, however, we propose a differential connection to the front end amplifier. This type of input connection to a SiPM has the advantage of superior rejection of ground and supplyvoltage noise, a key feature for a fast multichannel readout ASIC for TOF-PET applications where common mode noise can easily be transformed into electronic time jitter.

To evaluate this novel approach, we have used an ASIC already developed for the time of flight detector of the CERN ALICE experiment (NINO) [5]. This fast multichannel differential amplifier-discriminator works as a leading edge discriminator that also encodes, via time over threshold, the energy with the width of the digital output pulse. Connected to a time-based readout system $[6,7,8,9]$, it constitutes an extremely fast, compact and cost effective TOF-PET readout system, provided the ASIC integrates the complete processing channel. Moreover, this differential amplifier-discriminator, currently available in $0.25 \mu \mathrm{m}$ CMOS technology, could be designed in even deeper CMOS technology [10]. A large number of channels [11] can also be accommodated in such an ASIC together with the time-to-digital-converters TDCs [12].

The next section describes the principle of the differential current mode amplifier-discriminator and its connection to the SiPM; thereafter we present the Monte Carlo simulation results of photon production in LSO, as well as the SPICE circuit model of the SiPM together with simulation results of the entire channel. In Section IV we describe the experimental setup used to determine the time precision, and in Section V we present our experimental results and compare them with the simulations.

\section{FRONT-END AMPLIFIER DISCRIMINATOR (NINO)}

Several factors affect the timing performance of a PET detector: photon statistics, the photon transport, rise time of 
the crystal scintillation, the quantum efficiency of the photodetector and the avalanche timing uncertainty of the SiPM. In contrast to these, the timing limitations of the amplifier-discriminator circuit are far less severe, and therefore the amplifier-discriminator performance is more easily optimized for time jitter, being substantially lower than that produced by the scintillator and the SiPM. It is in fact feasible to set the discriminator threshold as close as to the detection of a single photon so as to minimize the jitter provoked by photon statistics alone. Each readout channel of the SiPM is then made of two functionally different circuits (see Fig. 1): First, the SiPM's anode and cathode are both connected to the inputs of the differential current mode amplifier stage, which is in turn followed by a leading edge discriminator stage employing time-over-threshold processing. The digital output pulse with its leading edge provides the time stamp, which if necessary could be corrected for time walk via the output pulse width, while the pulse width on its own is used to encode the photon energy.

\section{A. Differential time-based readout technique}

The time-based readout system, encoding photon energy with the width of the discriminator pulse, also offers the advantage of filtering, i.e. directly at the output of the discriminator stage, the acquired events for those contained in the photoelectric peak $(350-550 \mathrm{keV})$. This can be achieved with a simple digital circuit that vetoes the discriminator output for low energy photon signals (e.g. Compton scattered) and SiPM dark noise. This feature is particularly important when the discriminator threshold is set to a value close to signal height of one single photon where background is expected to be large.

Referring to Fig. 1 the differential connection between the SiPM and the amplifier inputs is obtained by adding a series load resistor to the cathode and anode terminals where the anode is referenced to ground. In this scheme the SiPM is connected to the readout system based on a concept previously developed and successfully implemented in the TOF-MRPC ${ }^{1}$ detector of the ALICE Experiment having a timing precision of $\leq 25 \mathrm{ps} r m s$. The $\mathrm{LVDS}^{2}$ discriminator outputs are then connected to a TDC completing the time-based readout architecture [13].

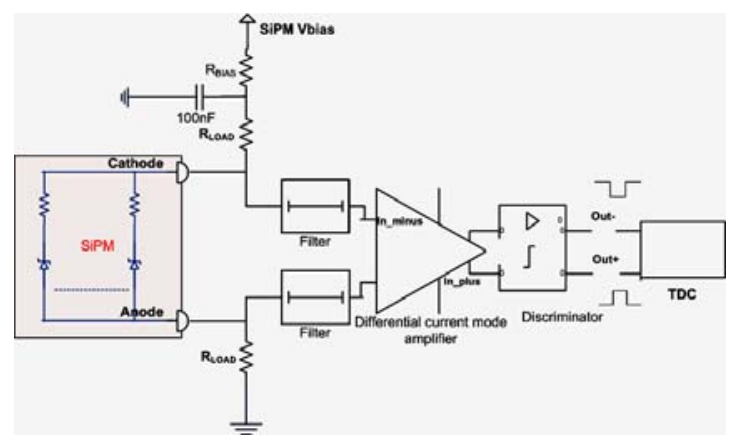

Fig. 1 Principle of the differential time-based readout. The circuit has a differential configuration from the SiPM terminals to the input of the TDC.

\footnotetext{
${ }^{1}$ MRPC stands for Multiple Resistive Plate Chambers

${ }^{2}$ LVDS is Low Voltage Differential Signal
}

This fully differential system approach offers superior common mode rejection and thus much better noise immunity, together with compact signal processing.

\section{B. Optimization of the input stage amplifier with the SiPM}

The preamplifier stage is optimized for single photon event sensitivity in order to detect the very beginning of a signal delivered by the LSO crystal. The optimization is done on an existing differential amplifier-discriminator ASIC (NINO), described in detail elsewhere [5]. It is based on a common gate input transistor pair with a differential configuration to sense the unbalanced current produced by the input signal. At the input the circuit can work with a single ended configuration or with a differential connection to the SiPM with the other negative input referred to ground. Fig. 2 shows the simplified equivalent circuit of the amplifier and SiPM for a single input circuit configuration to assess circuit optimization.

The simplified SiPM-amplifier circuit of Fig. 2, without the cascade stages and additional current sources, comprises the capacitance of a single SPAD, $C_{S P A D}$, transferring the SPAD charge to the total capacitance of $n \times C_{S P A D}$ of the SiPM terminal when an avalanche occurs. $C_{S P A D}$ is the equivalent circuit of the junction capacitance, and $n$ is the number of SPAD cells of the SiPM; parasitic capacitances are neglected. Therefore the signal voltage at the input of the transconductance preamplifier triggered by a single SPAD avalanche is:

$$
V_{I N} \sim\left(V_{\text {Bias }}-V_{\text {Avalanche }}\right) / n
$$

Taking $R_{S}$ as the drain resistor of the input transistor that senses the current unbalance in the two input branches the following signal is delivered to the second stage:

$$
\Delta V_{S} \sim \operatorname{gms} \times R_{S}\left(V_{\text {Bias }}-V_{\text {Avalanche }}\right) / n
$$

In case of the Hamamatsu MPPC with 3600 SPAD cells and a typical over-bias of $1.5 \mathrm{~V}$, the input voltage signal of the preamplifier is about $400 \mu \mathrm{V}$ for a single photon (one fired pixel SPAD). Sensing such a low signal on a large capacitance of $320 \mathrm{pF}$, which is typical for $3 \times 3 \mathrm{~mm}^{2}$ devices like the Hamamatsu S10931-33-50P, requires a careful amplifier design. The input transistor should be dimensioned with large transconductance, for this maximizes the amplifier's sensitivity and at the same time minimizes series noise. The chosen source transconductance gms of $50 \mathrm{mS}$ lowers the amplifier input resistance to $20 \Omega$, thought to be sufficient for a tolerable minimum of the input signal time constant of the SiPM. The Equivalent Noise Charge ENC, with the SiPM connected to the amplifier, is about $5 \mathrm{fC}$ rms with an amplifier peaking time of $1.5 \mathrm{~ns}$. For a single pixel fired, the jitter would be about 100 ps for a signal-to-noise ratio of 15 and a rise time of $1.5 \mathrm{~ns}$. The noise that makes up the aforementioned electronic time jitter of $\geq 25 \mathrm{ps}$ is for a large signal, a value significantly lower than that from the LSO crystal and SiPM. The amplifier input stage is not linear, saturating for an input current of about $250 \mu \mathrm{A}$, whereas at $511 \mathrm{keV}$ photon-energy the SiPM current reaches a peak value of $\sim 3 \mathrm{~mA}$. This feature enhances channel sensitivity at low discriminator thresholds 
by providing a larger gain at the very beginning of the SiPM signal.

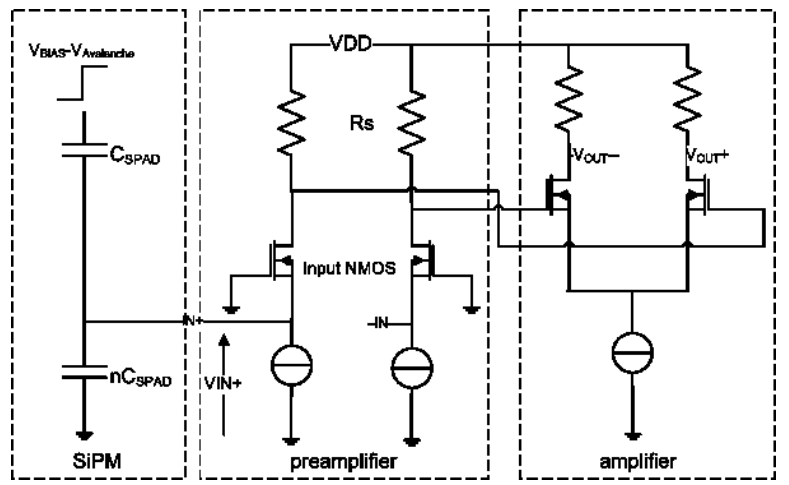

Fig. 2. Circuit principle of the differential amplifier input stage. For simplicity, cascade stages, additional current sources and DC stabilization are not drawn.

Hence the non-linearity of the input amplifier functions as a time-over-threshold signal processor before the discriminator stage sets in. To optimize the time-over-threshold response with a SiPM signal a passive differential filter has been inserted between the SiPM terminals and the amplifier inputs.

\section{Simulation Of The TOF-PET CHANNEL}

The timing precision of a PET detector is dominated by the time spread due to the intrinsic photon emission process [14] and the photon transport in the scintillator. Another limitation comes from the scintillation rise time in the emission process which might be estimated between 0.1 to $0.5 \mathrm{~ns}$ for a crystal made of LSO [15]. This latter effect has not been implemented yet in our simulation model. Our model so far includes all components of the detector channel, from the scintillation crystal (LSO) to the output of the discriminator. Photon emission statistics are dealt with via Monte Carlo simulation on one hand while the electronic effects in the chain, from the SiPM to the discriminator, are treated with SPICE. As such, the output of the Monte Carlo simulation is fed as input into the SPICE circuit model.

\section{A. Monte Carlo Simulation of light production in LSO}

In Fig. 3 we show the results of a Monte Carlo simulation carried out with Geant 4 [16,17] for a LSO crystal of $3 \times 3 \times 15 \mathrm{~mm}^{3}$ oriented along the Line of Response (LOR) in a PET detector. It shows the fluctuation in the arrival time of the $5^{\text {th }}$ photon collected by the SiPM. The most important figures which limit the time resolution and which are taken into account here, are the light yield, the decay time and the spread in the depth of interaction of the incident gamma-ray. The main simulation parameters are shown in Table I. Both, the wavelength dependent photon detection efficiency of the SiPM [18] and the LSO emission spectrum are simulated.

Table I. Monte Carlo simulation parameters

\begin{tabular}{|l|l|l|l|l|}
\hline $\begin{array}{l}\text { LIGHT } \\
\text { YIELD } \\
{\left[\mathrm{MEV}^{-1}\right]}\end{array}$ & $\begin{array}{l}\text { DECAY } \\
\text { TIME } \\
{[\mathrm{NS}]}\end{array}$ & $\begin{array}{l}\text { RESOLUTION } \\
\text { SCALE }\end{array}$ & $\begin{array}{l}\text { REFRACTIVE } \\
\text { INDEX }\end{array}$ & $\begin{array}{l}\text { ABSORPTION } \\
\text { LENGTH } \\
{[\mathrm{CM}]}\end{array}$ \\
\hline 23000 & 40 & 4.41 & 1.82 & 13.8 \\
\hline
\end{tabular}

Under these assumptions the fluctuation $5^{\text {th }}$ photon arrival time is $77 \mathrm{ps} r m s$, seen in a single detector, corresponding to a coincidence time precision of $\geq 250 \mathrm{ps} F W H M$. Consequently, in the case of the $1^{\text {st }}$ photon to be detected, not shown here, the coincidence timing fluctuation would improve to $122 \mathrm{ps}$ FWHM. This sets a lower limit to the achievable time precision in a TOF-PET system, for it effectively is the irreducible, physical limit imposed by the LSO crystal of the tested size of $3 \times 3 \times 15 \mathrm{~mm}^{3}$ when exposed to $511 \mathrm{keV}$ gamma rays. The result clearly shows that the ideal detection threshold should be at the level of one photon, which on the other hand in view of the SiPMs dark noise is rather difficult to achieve.

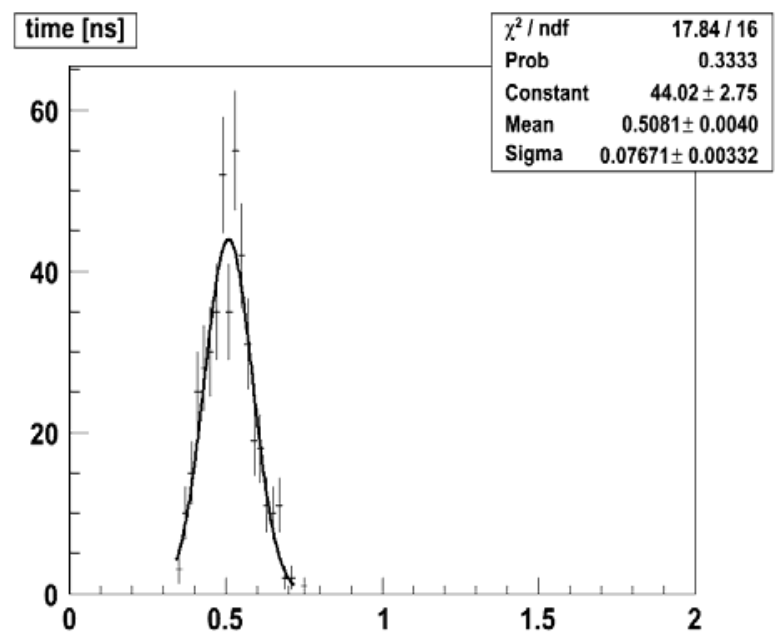

Fig. 3. Arrival time fluctuation of the $5^{\text {th }}$ photon. The simulation includes photon statistics and the effects of photon transport in the LSO crystal.

\section{B. Electronic (SPICE) modeling of the SiPM}

The precise electrical modeling of the SiPM is an important ingredient in the understanding of the timing performance of the complete PET detector chain. We have chosen to model the Hamamatsu MPPC S10362-33-050c with CADENCE HSPICE assuming an electrical circuit to be as close as possible to the physical device, based on the Cova model [19]. The equivalent circuit of a SPAD cell is shown in Fig. 4. It comprises four functionally important blocks, i.e. the SPAD diode core, the quenching resistor, the avalanche trigger generator and the interconnect capacitance. The complete SiPM-SPICE model then consists of an array of 3600 such SPAD circuits interconnected with each other in parallel.

To 'generate' an avalanche a time dependent resistance, acting as a switch, is used in each SPAD cell. In SPICE the avalanche time is a hierarchical parameter so as to individually feed each of the 3600 SPAD cells of the SiPM with the photon time sequence generated before by the Monte Carlo simulation as explained in Section III.A. The relevant SPICE parameters and their values used for the MPPC (Hamamatsu S10362-33050c) are shown in Table II. Parameters have been estimated from the pulse shape of the single photon signal response. Note, from our comparison of the single-photon-SPICE results with the experimental results (see Section VII), some of the key parameters in the model, 


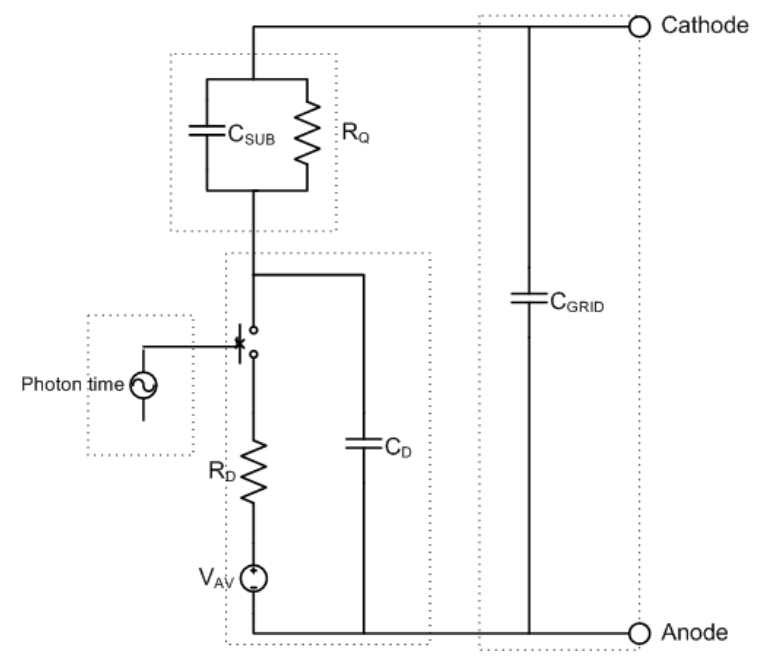

Fig. 4. SPICE model of the SPAD cell. Simulation includes 3600 cells connected in parallel.

such as the experimental rise time, the signal amplitude and decay time, due to their uncertainties, had to be adjusted to achieve optimum conformity with the data. For example, we have observed that a series inductance (not shown) in the SPAD diode circuit (see Fig. 4) significantly influences the timing behavior of the circuit to the extent that it may override the effect of the parameterized resistor $\mathrm{R}_{\mathrm{D}}$ in the model.

TABLE II. VALUES OF THE SIPM PARAMETERS OF THE SPICE MODEL

\begin{tabular}{|c|c|c|c|c|c|c|}
\hline$R_{D}[\Omega]$ & $V_{A V}[V]$ & $V_{O V}[V]$ & $C_{S P A D}[f F]$ & $R_{Q}[\Omega]$ & $C_{G R I D}[f F]$ & $C_{S U B}[f F]$ \\
\hline $1 \mathrm{k}$ & 69.5 & 1.5 & 65.5 & $30 \mathrm{M}$ & 16 & 50 \\
\hline
\end{tabular}

$\mathrm{C}_{\mathrm{SPAD}}$ is the effective SPAD capacitance, $\mathrm{R}_{\mathrm{D}}$ the junction series resistance, $\mathrm{R}_{\mathrm{Q}}$ the bias resistance, $\mathrm{C}_{\mathrm{SUB}}$ the substrate capacitance, $\mathrm{C}_{\mathrm{GRID}}$ the interconnect capacitance, $\mathrm{V}_{\mathrm{AV}}$ the avalanche voltage, and $\mathrm{V}_{\mathrm{OV}}$ the over-bias voltage.

\section{Simulation results of the Entire Readout Channel}

For a single photon event the unbalanced current signal at the differential input ports of the amplifier is $20 \mu \mathrm{A}$. Then the voltage signal across the $2.5 \mathrm{k} \Omega$ sensing resistors $\mathrm{R}_{\mathrm{S}}$ (see Fig. 2) is $50 \mathrm{mV}$, a sufficient signal to detect a single photon. Fig. 5 shows the simulation results obtained for four different bias conditions and with the discriminator threshold set to the minimum, i.e. $50 \mathrm{mV}$.

When $511 \mathrm{keV}$ gamma rays illuminate the LSO crystal typically $\sim 2000$ photons are impinging on the SiPM over a duration, i.e. an exponential signal fall time $\tau$, of $40 \mathrm{~ns}$. Single photon signals are then piling up to each other and form the signals shown in Fig. 6. The corresponding discriminator pulse widths vary with the SiPM current signal, e.g. from 20ns for an input signal equivalent to 100 photons to $120 \mathrm{~ns}$ for a 2000-photon-signal. As we had indicated in Section II.B and expected from the time-over-threshold stage, the discriminator response is nonlinear. However, this nonlinear response has advantages that it encodes signals with a very large dynamic range, i.e. from 1 to 2000 photons, and this in a short time and via a very simple hardware processing, offering in addition the possibility of selecting events in the energy window around the photoelectric peak.

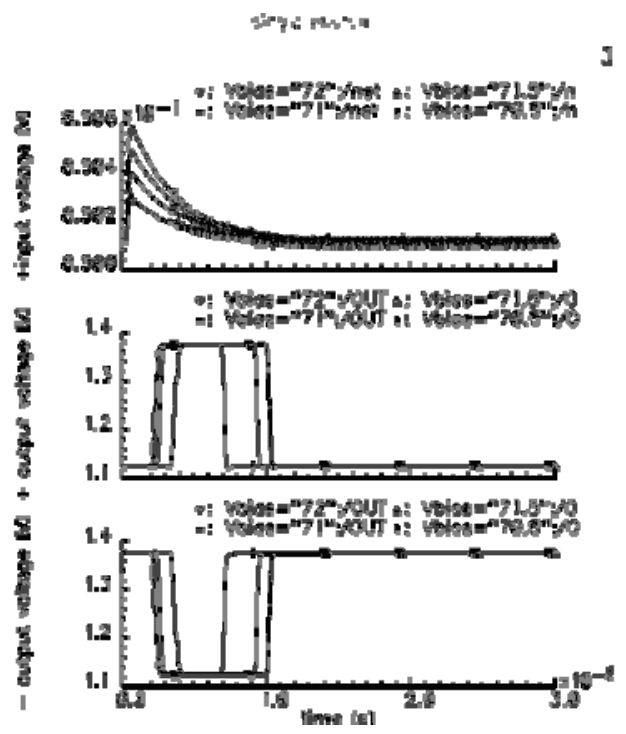

Fig. 5. Single photon SPICE simulation of the discriminator with the SiPM connected for four over-bias voltages between $1 \mathrm{~V}$ and $2.5 \mathrm{~V}$. Amplifier input amplitudes (top graph) range from $200 \mu \mathrm{V}$ (@ 1V over-bias) to $500 \mu \mathrm{V}(2.5 \mathrm{~V}$ over-bias). The corresponding pulse widths of the discriminator (bottom graphs) then vary between $3 \mathrm{~ns}$ and $8 \mathrm{~ns}$.

Owing to this characteristic an important question is, how many initial photons are needed to operate the amplifierdiscriminator at stable signal discrimination. For $511 \mathrm{keV}$, for example, LSO provides a typical signal of in average 50 photons within the first ns.

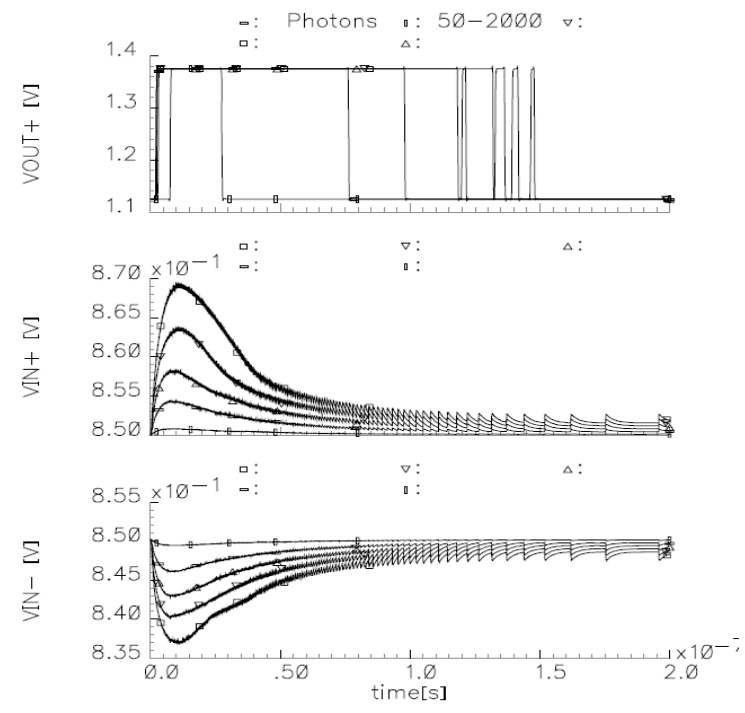

Fig. 6. SPICE simulation result of the amplifier discriminator response for a signal ranging from 100 photons to 2000 photons. Top signal is the discriminator output, the bottom two signals are the signal seen at the differential amplifier inputs. For this simulation, the SiPM SPICE model is driven by packets of 10 photons.

To estimate the channel sensitivity to the first photons arriving at the detector input, our Monte Carlo model (Section III.A) generated a random time sequence of photons which served as input to the SiPM-SPICE model. Simulations have been carried out from as low as one photon up to the saturation point of the amplifier input stage which is at $\geq 10$ 
photons. The corresponding time walk in signal response was also obtained from these simulations and is shown in Table III.

TABLE III. SIMULATED TIME WALK FOR THE FIRST ARRIVING PHOTONS OF A LSO SIGNAL WITH 2000 PHOTONS IN TOTAL. OVERBIAS OF THE SIPM IS SET TO $1.5 \mathrm{~V}$.

\begin{tabular}{|l|l|l|l|l|l|l|l|l|}
\hline \# Photon & 1 & 2 & 3 & 4 & 5 & 6 & 7 & 8 \\
\hline walk [ns] & 1.8 & 1.52 & 1.91 & 1.35 & 1.30 & 1.29 & 1.29 & 1.29 \\
\hline
\end{tabular}

This demonstrates that single photon detection is feasible with proper timing correction and, that above 6 photons, time walk is constant.

We have also estimated (via Monte Carlo) the LSO inherent time jitter for two cases of 10 LSO photons arriving at the detector over two different time sequences and hence producing a time shift in the discriminator response. Under these circumstances a FWHM jitter of about 99 ps is observed (Fig. 7).

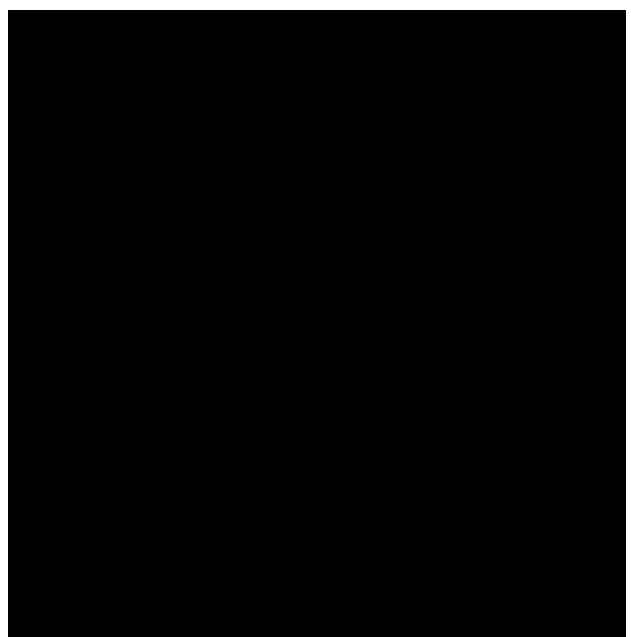

Fig. 7. Jitter simulation for two timing sequences of the first 10 photons Jitter induced by photon statistics can be estimated to FWHM of 99ps. SiPM over-bias is $1.5 \mathrm{~V}$.

\section{LASER MEASUREMENTS WITH SiPM}

\section{A. Laser timing and calibration}

We use a $405 \mathrm{~nm}$ pulsed laser from PicoQuant with a pulse width of 50ps FHWM. The laser is operated at very low optical power to illuminate the SiPM (Hamamatsu S10362-33050c) with few photons only. Bias conditions of the SiPM are identical to the ones treated in the SPICE circuit model. Pulse amplitude, pulse rise and decay time are recorded with a digital oscilloscope. Both the anode and cathode terminals of the SiPM are connected to the differential amplifierdiscriminator, exactly as it was simulated with the SPICE model. Fig. 8 right shows the delay time jitter measured between the laser gate, acting as a trigger, and the discriminator output as a function of the width of the NINO output. The cluster of single photon events are highlighted by a line, and projected to the delay time Y-axis show a jitter of 260 ps. A fit of the multi-photon data have been done and indicates that time jitter improves statistically like $1 / \sqrt{N}$, with $\mathrm{N}$ photons hitting the SiPM.

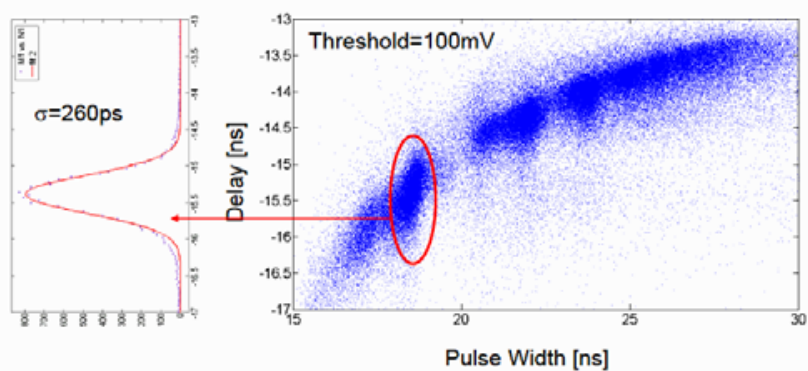

Fig. 8 Time-delay scatter plot for few photons from the laser illuminating the MPPC. Single photon time jitter is about 260 ps.

\section{V.COINCIDENCE MEASUREMENTS WITH ${ }^{22}$ NA}

\section{A. Experimental setup}

As shown in Fig. 9, a $4 \mathrm{MBq}^{22} \mathrm{Na}$ source is placed between two detectors in coincidence. The first detector, a Hamamatsu PMT H6533, serves as reference and is coupled to a LSO crystal of $2 \times 2 \times 10 \mathrm{~mm}^{3}$ size with a light yield of $17^{\prime} 800$ photons $/ \mathrm{MeV}$ in vertical position. LSO crystals are wrapped with Teflon. The PMT output is connected to a constant fraction discriminator (CFD). The opposite detector, a Hamamatsu SiPM or MPPC S10362-33-050C, is connected to a LSO crystal of $3 \times 3 \times 15 \mathrm{~mm}^{3}$ size and a light yield of $21^{\prime} 000$ photons $/ \mathrm{MeV}$. The output of the SiPM is connected differentially as described before to the NINO amplifierdiscriminator chip. All crystals are coupled with silicon grease to their photo detectors. Both detector systems are at a distance of $\sim 2 \mathrm{~cm}$ from the ${ }^{22} \mathrm{Na}$ source and read out with a digital oscilloscope LeCroy WavePro 7100.

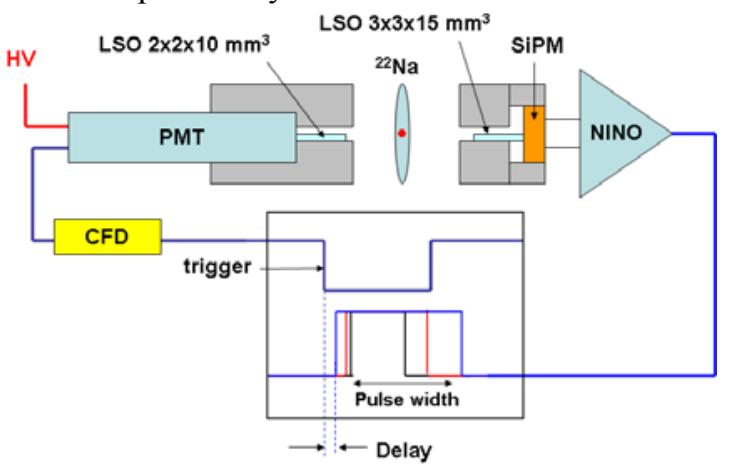

Fig. 9 Principle of the experimental setup, showing left the PMT and to the right the MPPC readout with the amplifier-discriminator ASIC NINO.

\section{B. Measurements and results}

In a previous (not shown here) reference timing measurement of a PMT vs. PMT (both Hamamatsu H6533 and coupled to LSO of $2 \times 2 \times 10 \mathrm{~mm}^{3}$ ) a coincidence time resolution of 362ps FWHM was found. This leads to the intrinsic time resolution of $362 / \sqrt{2}=256$ ps FWHM of one PMT. For the measurement of a PMT vs. a SiPM, both the photon energy, i.e. given by the NINO pulse width, and the time information, i.e. the delay between the CFD's and NINO's outputs, were recorded and entered into a scatter-plot "Delay vs. Width". This is shown in Fig. 9 graph (2). The projection of the data entries on the coordinate axes unfolds (3) the energy spectrum 
of the detector and (1) the time histogram of the signal delay between the coincident detectors. In this measurement, the MPPC was biased at $71.5 \mathrm{~V}(2 \mathrm{~V}$ above the breakdown voltage of $69.5 \mathrm{~V})$.
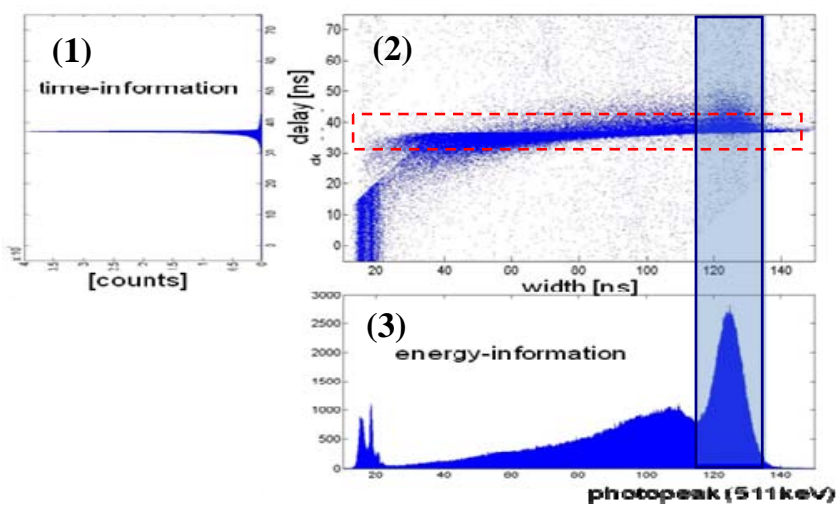

Fig. 10 Scatter-plot of the delay and pulse width (energy) spectrum of the PMT vs. the Hamamatsu MPPC. The projections denote the energy spectrum of ${ }^{22} \mathrm{Na}$ (horizontal) and the coincidence timing distribution (vertical). The first peak is the single avalanche coming from the MPPC dark rate.

Events between $33 \mathrm{~ns}$ and $43 \mathrm{~ns}$ delay (dashed horizontal rectangle) in Fig. 10 (2) are considered true coincidence events. In this region the delay variation in the histogram changes as a function of pulse width for it is being influenced by time walk. This is an 'inbuilt' consequence of the timeover-threshold technique used here. The effect of this is that the delay histogram widens, as seen in Fig. 10 (1), and that a proper correction should be done prior to deriving the ultimate time resolution. Also the clear presence of the ${ }^{22} \mathrm{Na}$ photopeak in Fig. 10 (3) allows yet another improvement in time resolution, by selecting only (vertical rectangle) events within $\pm 3 \sigma-6 \sigma$ around the mean of this peak, corresponding to approximately $110 \mathrm{~ns}-140 \mathrm{~ns}$ in pulse width. MPPC single avalanche from thermal noise seen between 0ns and $20 \mathrm{~ns}$ equivalent pulse width has been crosschecked with the single photon peak obtained in Fig. 8.

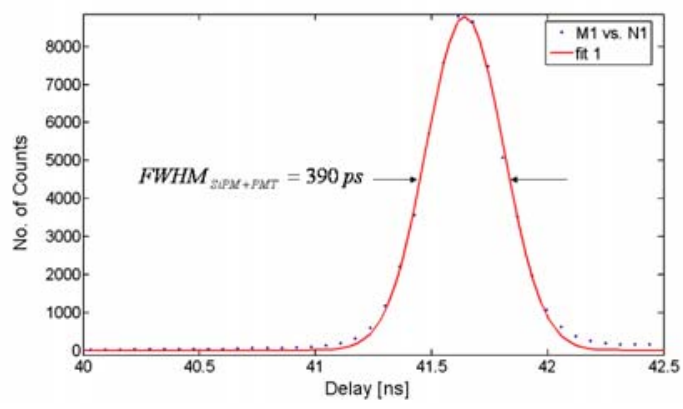

Fig. 11. Time coincidence distribution of SiPM vs. PMT after time walk correction and photopeak selection FWHM=390 ps

As we had stipulated in Section II.B the pulse width histogram, because of the time over threshold technique used in NINO, is not linear with charge or energy. Also the fact that dark rate thermal noise is visible in the spectrum indicates that the NINO threshold is set between 1 and 2 single photon threshold, i.e. at the level of one-photon detection. Applying time walk correction and photopeak-event selection, the time resolution of the combined PMT-SiPM system is 390 ps time coincidence FWHM (see Fig. 11).

The intrinsic single SiPM-based time resolution is then derived from

$$
\begin{aligned}
& F W H M_{S i P M+P M T}=\sqrt{F W H M_{S i P M}^{2}+F W H M_{P M T}^{2}}=390 p s \\
& F W H M_{S i P M}=\sqrt{390^{2}-275^{2}}=277 p s
\end{aligned}
$$

In a coincident setup made of two MPPCs, the system coincidence time resolution $C T R$ becomes:

$$
C T R=\sqrt{2} \times 277=392 p s
$$

\section{INTERPRETATION AND DISCUSSION OF RESULTS}

The contributions on time resolution from the different elements in the detector system have been studied in detail in [20,21].

Photon production statistics in the scintillator alone produce a timing uncertainty $\sigma_{j}$ phot

$$
\sigma_{j p h o t}=\tau \cdot \ln \left(\frac{1}{2}\left[Q-R+\sqrt{(Q-R)^{2}+4 R}\right]\right)
$$

where $R$ denotes the total number of photons detected, $\tau$ the LSO-signal decay time constant of 40ns, and $Q$ the photon number on which the NINO threshold is set.

Time uncertainty due to the photon transit path in the scintillator is expressed as $\sigma_{j}$ transit

$$
\sigma_{j \text { transit }}=\frac{2 L n}{c\left|\cos \left(\theta_{\max }\right)\right|}
$$

with $L$ being the scintillator length, $n$ the index of refraction of LSO (1.85), $c$ the speed of light in vacuum and $\theta_{\max }$ the angle for which the transit time of a photon in the LSO crystal is maximum(in our set up $\theta_{\max }$ is $131^{\circ}$ ).

Time jitter due to electronics noise is expressed as $\sigma_{j}$ :

$$
\sigma_{j}=\sigma_{e} /\left(\frac{d V}{d t}\right)_{\text {threshold }}
$$

where $\sigma_{e}$ denotes electronics noise, and $(d V / d t)_{\text {threshold }}$ the slope of the SiPM signal at the NINO threshold crossing.

The total jitter can then be written as $\sigma_{j \text { total }}$ :

$$
\sigma_{j \text { total }}=\sqrt{\sigma_{j \text { phot }}^{2}+\sigma_{j \text { transit }}^{2}+\sigma_{j}^{2}}
$$

Since in equation (3) both $R$ and $\tau$ are constant the minimum time uncertainty due to photon statistics alone is reached with $\mathrm{Q}=1$, thus setting the $\mathrm{NINO}$ discriminator threshold at the level of a single photon to be detected.

To minimize transit time and associated jitter in the crystal one would obviously want to choose short crystals as the time jitter is directly proportional to crystal length (equation (4)). This gain, however, must be weighed against a rapid loss in detection efficiency with decreasing crystal size. 
Finally it is deduced from equation (5), that the electronics front end needs to be carefully designed to keep noise sources at a minimum and yet preserve the fastest ballistic response $(\mathrm{dV} / \mathrm{dt})$ of the signals present at the input.

A comparison of the Monte Carlo/SPICE results with our measurements is shown in Table IV.

TABLE IV. COMPARISON OF MONTE CARLO/SPICE RESULTS WITH MEASUREMENTS

\begin{tabular}{|c|c|c|}
\hline $\begin{array}{c}\text { Time jitter as predicted by } \\
\text { Monte Carlo + SPICE: }\end{array}$ & $\begin{array}{c}\text { Measured time jitter } \\
\text { from laser tests: }\end{array}$ & $\begin{array}{c}\text { Measured time jitter } \\
\text { from }{ }^{22} \text { Na tests: }\end{array}$ \\
\hline 148ps FWHM & $270 \mathrm{ps} \mathrm{FWHM}$ & $390 \mathrm{ps}$ FWHM \\
\hline
\end{tabular}

The discrepancy between model predictions and test results is still rather large. Several issues, at this stage of our investigations not yet entirely clarified, would most probably contribute to this inconsistency:

\section{1) Crystal:}

It seems that the models are too optimistic in terms of photon statistics, i.e. predicting the correct number of photons illuminating the photo detector. In other words, the quality of the measurements may have suffered from imperfect optical coupling between the crystal and the SiPM. Likewise, the simulations may have overestimated parameters like crystal-face reflectivity and perhaps other optical effects in the crystal. As we had stated before, the scintillation rise time has not yet been introduced as a modeling parameter in these simulations either.

\section{2) Photo detector (SiPM)}

SPICE may have misjudged the rise time of the SiPM signal development, probably taken shorter than the measured one. SiPM noise has not been included in the simulation, and with a frequency rate of about $10 \mathrm{Mhz}$, it substantially degrades the jitter figure.

\section{3) Electronics}

In modeling the noise behavior of the electronics SPICE had used a simplified algorithm perhaps not yielding the correct result. On the other hand, a measurement of the electronic noise in the presence of an input capacitance of $320 \mathrm{pF}$ is still outstanding, too, for a better understanding of the front end behavior.

\section{SUMMARY}

From the fact that the time jitter obtained from laser illumination tests on the SiPM (no crystal present) is substantially lower than that derived from the ${ }^{22} \mathrm{Na}$ coincidence measurements, lead us to conclude that most of the discrepancy between model and data stems from effects in the crystal and the photo detector and to a lesser degree from electronics. Further measurements will clarify these issues in the near future.

Another important outcome of this work is an estimate of the lower limit to the timing performance of a TOF-PET system. Simulation shows a limit of about 150ps FWHM in coincidence time precision for a crystal like LSO and a size of $3 \times 3 \times 15 \mathrm{~mm}^{3}$. Therefore it appears rather unlikely that the ambitious goal of 100ps FWHM time resolution could be obtained in current (long) LSO scintillators without significant loss in efficiency when shortening the crystals for the sake of better timing. A large effort in developing faster crystals with an increased photon yield as well as photo detectors with higher quantum efficiency over present devices may ultimately achieve this goal.

\section{VIII.REFERENCES}

[1] Joel S. Karp et al. Benefit of Time-of-Flight in PET: Experimental and Clinical Results, J Nucl Med Karp et al. 49 (3), 462.

[2] C.L. Kim, G.C. Wang, S. Dolinsky, Multi-Pixel Photon Counters for TOF-PET Detector and its challenges, 2008 IEEE NSS symposium

[3] C. de La Taille et al. SPIROC. 1857. IEEE NSS conference record, 2007, Vol. N29-5.

[4] F. Corsi et. al, ASIC development for SiPM readout Pixel 2008 international workshop Batavia : Jinst IOP, 2009R.

[5] P. Jarron et al. NINO: an ultra-fast and low-power front-end amplifier/discriminator ASIC designed for the multigap resistive plate chamber, NIM A, 2004, Vol. 533 page 183-187.

[6] A Time-Based Front End Readout System for PET \& CT ,Meyer, T.C.; Powolny, F.; Anghinolfi, F.; Auffray, E.; Dosanjh, M.; Hillemanns, H.; Hoffmann, H.-F.; Jarron, P.; Kaplon, J.; Kronberger, M.; Lecoq, P.; Moraes, D.; Trummer, J.; Nuclear Science Symposium Conference Record, 2006. IEEE Volume 4, Oct. 29 2006-Nov. 1 2006 Page(s):2494 - 2498.

[7] A Novel Time-Based Readout Scheme for a Combined PET-CT Detector Using APDs, Powolny, F.; Auffray, E.; Hillemanns, H.; Jarron, P.; Lecoq, P.; Meyer, T.C.; Moraes, D.; Nuclear Science, IEEE Transactions on Volume 55, Issue 5, Part 1, Oct. 2008 Page(s):2465 - 2474.

[8] F. Anghinolfi, J. Christiansen, P. Jarron. Readout circuit for use in combined PET CT apparatus. WO2008 040384 international patent, 02 November 2006. PCT/EP2006/010524.

[9] J. Christiansen et al. "A flexible multi-channel high resolution time to digital converter ASIC", Nuclear Science Symposium and Medical Imaging Conference : NSS/MIC 2000, Lyon - France, 16 - 20 Oct. 2000.

[10] M. Despeisse, P. Jarron, F. Anghinolfi, S. Tiuraniemi, F. Osmic, P. Riedler, A. Kluge, A. Ceccucci, Nuclear Science, IEEE Transactions on Volume 56, Issue 2, April 2009 Page(s):375 - 381.

[11] J. Lapington, M. Despeisse, et al, "A multi-channel high-time resolution detector for high-content imaging", article in press Nucl. Instr. And Meth. A, 2009

[12] E. Martin et al, the 5ns Peaking Time Transimpedance Front End Amplifier for the Silicon Pixel Detector in the NA62 Experiment presented in this conference.

[13] A. Akindinov et al. Final test of the MRPC production for the ALICE TOF detector, NIM A, Volume 602, Issue 3, 1 May 2009, Pages 709712.

[14] M. Kelbert, I. Sazonov, A.G. Wright, Exact expression for the variance of the photon emission process in scintillation counters, NIM A 564 (2006) 185-189

[15] Y. Shao, A new timing model for calculating the intrinsic timing resolution of a scintillator detector, Phys.Med. Bio. 52 (2007) 11031117.

[16] J. Allison et al., Geant4 Developments and Applications, IEEE Transactions on Nuclear Science 53 No. 1 (2006) 270-278

[17] S. Agostinelli et al., Geant4 - A Simulation Toolkit, Nuclear Instruments and Methods A 506 (2003) 250-303.

[18] Hamamatsu Photonics, MPPC data sheet, www.hamamatsu.com

[19] S. Cova et al., Avalanche Photodiodes and Quenching Circuits for Single-photon Detection, Applied Optics, pp. 1956-1976, Vol. 35, N. 12, 1996

[20] F. Powolny et al. A Time Driven Readout Scheme for PET and CT Using APDs and SiPMs in press NIM A

[21] F.Powolny, Characterization of time resolved photodetector systems for PET. PhD thesis http://doc.rero.ch/record/12683?ln=de 